Chem. Eny. Comm. 1996, Vols. 141 142, pp. 155173 Reprints available directly from the publisher Photocopying permitted by license only
(19) 1996 OPA (Overseas Publishers Association)

Amsterdam B.V. Published in The Netherlands under license by Gordon and Breach Science

Printed in Malaysia

\title{
BUBBLE AND SLUG FLOW AT MICROGRAVITY NL CONDITIONS: STATE OF KNOWLEDGE AND OPEN QUESTIONS
}

\author{
C. COLIN and J. FABRE \\ Institut de Mécanique des Fluides de Toulouse, URA CNRS 005 , \\ Avenue Camille Soula, 31400 Toulouse, France \\ 03240
}

\section{J. MCQUILLEN}

National Aeronautics and Space Administration Lewis Research Center. Cleveland, Ohio 44135

(Received in final form July 3. 1995)

Based on the experiments carried out over the past decade at microgravity conditions, an overview of our current knowledge of bubbly and slug flows is presented. The transition from bubble to slug flow. the void fraction and the pressure drop are discussed from the data collected in the literature. The transition from bubble to slug flow may be predicted by introducing a critical void fraction that depends on the fluid properties and the pipe diameter: however, the role of coalescence which controls this trapsition is not clearly understood. The void fraction may be accurately calculated using a drift-flux modele thown from local measurements that the drift of the gas with respect to the mixture is due to the non unif, ilal distribution of void fraction. The pressure drop happens to be controlled by the liquid flow for slug flow the experimental results show that pressure drops is larger than expecteras study, the guidelines for future research in microgravity are given.

KEYWORDS Two phase flow Microgravity Bubble slug

\section{INTRODUCTION}

In the past decade several gas-liquid flow experiments have been conducted in microgravity conditions, using either free fall in drop tower facilities or parabolic flights in aircraft. Space applications, such as life support systems and thermal energy production and transport, have raised some classical technical problems that stimulated the development of two-phase flow research at microgravity conditions. Among these are the prediction of flow pattern, pressure drop, heat transfer and phase fraction in thermohydraulic systems. Beyond the design of space systems, reduced gravity two-phase flows can address some fundamental questions which remain unsolved. On earth, the force balance between the two phases is often dominated by the gravitational force. As the gravity is suppressed, a new balance comes into play between inertial, viscous and interfacial forces so that the mechanics which govern the interactions between phases drastically change. Low gravity condition are particularly favourable to the emergence of surface tension as dominating force whose role is often small at $1-\mathrm{g}$ conditions. 
In this paper we shall focus the discussion on bubbly and slug flowin tubes and to the related physical issues:

- What do we know about the transition between bubble and slug flow and what are the physical mechanisms to be understood?

- Is there a drift between phases, which forces are responsible for the drift and what are the future directions of research?

- Does the presence of the other phase modify the basic mechanism of wall friction and what are the unresolved questions?

These questions will be addressed in the following sections. We shall start from the state of knowledge that we have reached with the available experimental data and with some new data. These data were recently obtained in air-water flow in tubes of 6,10 and $19 \mathrm{~mm}$ diameters during parabolic flight experiments run in the "Zero-g" Caravelle aircraft. The experimental facility is the two-phase flow loop described by Colin and Fabre (1995): it is basically the same as the facility described by Colin et al.(1991).

\section{TRANSITION FROM BUBBLY TO SLUG FLOW}

Among the many experiments carried out in microgravity most are devoted to the interface morphology. Indeed the classification of two-phase flow by various patterns, although subjective, is easy to accomplish since it only requires a careful observation of the flow. In microgravity this observation is made from the pictures taken with high speed film or video camera. Several studies with and without phase change have been carried out over the past decade. They have been performed with various fluids, in tubes of different diameters $D$ and different lengths $L$ during the short periods of aircraft parabolic trajectories (between $10 \mathrm{~s}$ and $20 \mathrm{~s}$ depending upon the plane) or drop tower falls (about $2.2 \mathrm{~s}$ for the NASA Lewis Research Center's Drop Tower). The fluids used were either air and water, or boiling Refrigerant 12 or 114 . Some of the more recent studies are reported in Table I. Different flow pattern have been identified at different superficial velocities of liquid $j_{L}$ and gas $j_{G}$. Let us recall briefly the main results which have been put into light.

At low void fraction, bubbly flow occurs. At high superficial liquid velocity and low superficial gas velocity, small bubbles of a few millimetres appear. These bubbles are nearly spherical. Their motion is rectilinear with nearly the same velocity. The fluctuating motion is weak, contrary to what is observed in 1-g upward flow where bubbles of ellipsoidal shape rise with a fluctuating motion. The size of the bubbles is mainly controlled by coalescence as suggested by the evolution of the size distribution between the inlet and the outlet of the pipe (Colin et al., 1991). As the void fraction increases, larger bubbles are created. These bubbles move along the tube axis. When their size is comparable to the tube diameter, they take an oblate shape controlled by the tube size. Following the classification proposed by Dukler et al. (1988) the transition from bubbly to slug flow is defined when some bubbles with diameter larger than 1D or 2D appear. However, this transition is somewhat arbitrary since the larger bubbles grow with an increase in void fraction. There is no physical evidence of a pattern "bifurcation" (Colin et al., 1991). 


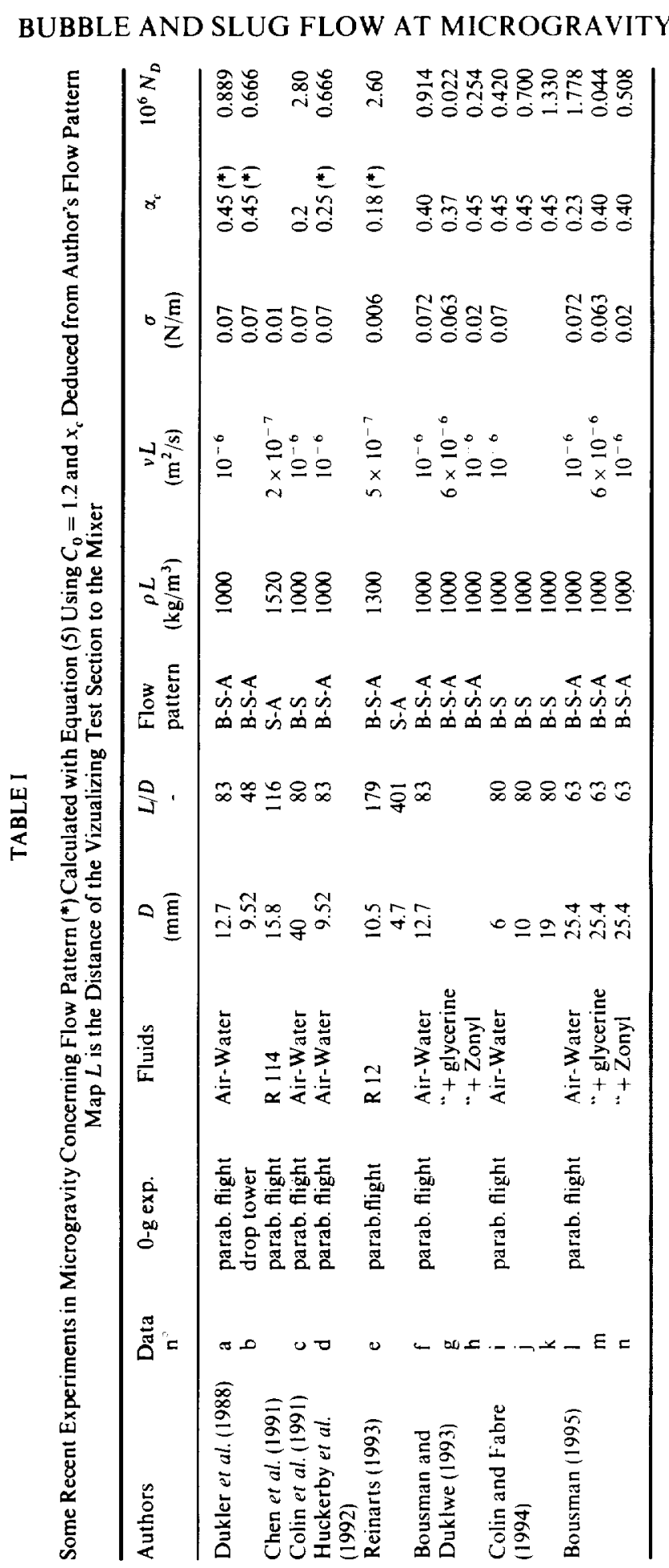


The large bubbles are the precursors of cylindrical bubbles that appear in slug flow at higher void fractions. These long bubbles which have a smooth interface and a spherical shaped nose, are separated by liquid slugs. These slugs contain smaller spherical bubbles moving nearly at the same velocity than the cylindrical bubbles. In contrast to 1-g upward flow these bubbles are not created by gas entrainment at the rear of the cylindrical bubbles: they are simply the residue of the initial bubbly flow which is injected at the inlet.

At the gas velocity increases, the liquid slugs decrease in length. When the liquid slugs are short enough they collapse. The resulting pattern consisting of lilquid flowing in the form of a film at the wall and gas flowing in the centre, is similar to annular flow. But the air core sometimes breaks up and frothy slugs, containing many small bubbles, appear. This flow pattern which is often called frothy slug-annular flow (Zhao and Rezkallah, 1993), is a transition between slug flow and annular flow, which occurs at the highest superficial velocities of the gas.

Let us focus on the transition between bubbly and slug flows. Flow pattern maps for bubbly and slug flow are shown in Figures 1,2 and 3 for different data sets, using the co-ordinates $j_{L}$ and $j_{G}$. Figure 1 is plotted with the data from Colin and Fabre (1995) for air-water flow in tubes of different size. Figure 2 is plotted with the data from Colin et al. (1991) for air-water flow in tube of $40 \mathrm{~mm}$. Figure 3 uses the data from Bousman (1995) for airswater flow.

Several authors have tried to predict the transition between bubble and slug flows, the arbitrary criterion being that the transition occurs when some bubbles in the flow reach a diameter equal to the tube diameter. The theories may be classified in three

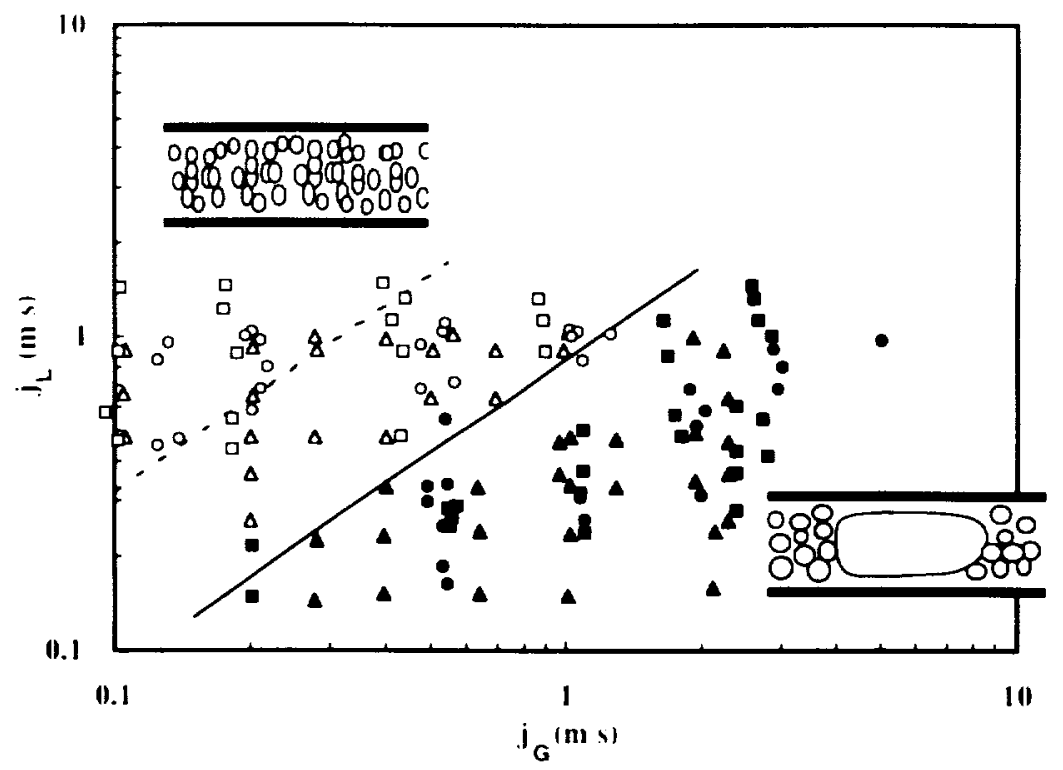

FIGURE $10-\mathrm{g}$ flow patterns in small tubes (Colin and Fabre, 1995): Data $i D=6 \mathrm{~mm}$ : obubble, $\bullet$ slug flows, Data $j D=10 \mathrm{~mm}: \square$ bubble, $\square$ slug flows, Data $k D=19 \mathrm{~mm}: \triangle$ bubble, $\triangle$ slug flows Transitions: bubbly to slug fiow: . Equation (2) $x_{c}=0.2 \ldots$ Equation (2) $x_{c}=0.45$. 


\section{REPERENCES}

Bousman. S. Studies of Two-Phase Gas-Liquid Flow in Microgravity, NASA Contractor Report 195434. (1995).

Bousman, S., and Duckler A. E. (December 1993) Studies of Gas-Liquid Flow in Microgravity: Void Fraction. Pressure Dron and Flow Pattern, Proceeding of the 1993 ASME Winter Meeting. New Orleans, LA. Fluid Mechanics in Microgravity Session. AMD-Vol. 174/F ED-Vol. 175. December (1993).

Bousman. S., and MacQuillen. J. Characterization of Annular Two-Phase Gas-Liquid Flows in Microgravity, Proce'edings of the NASA Second Microgratity Fluid Physic's Conference' NASA L_eRC. Cleveland, June (1994).

Chen. I. Y.. Downing, R. S., Keshock, F., and AI-Sharif, M. Measurements and Correlation of Two-Phase Pressure Drop under Microgravity Conditions. Journal of Thermophysics, 5, 514523 (1991).

Colin. C. Econdements diphasiques à hulle's et a poches en micrope'santew. These. INP Toulouse (1990).

Colin. C. Fabre, J., and Dukler, A. E. Gas-Liquid Flow at Microgravity Conditions-I: Dispersed Bubhle and Slug Flow, Int. J. Multiphase' Flom, 17, 533544 (1991).

Colin. C., and Fabre. J. Gas-liquid Pipe Flow Under Microgravity Conditions: Influence of Tube Diameter on Flow Patterns and Pressure Drops, submitted to Adr. Space Res (1995).

Collins, R., De Moraes. F. F. Davidson, J. F., and Harrison, D. The Motion of Large Bubbles Rising Through L.iquid Flowing in a Tube. J. Fluid Mech. 89. 497514 (1978).

Dukler. A. E.. and Fabre, J. Gas Liquid Slug Flow: Knots and Loose ends. Third Imernational Workshop om Two-Phase Flow Fundamentals, June 15 19. London. UK (1992).

Dukler, A. E., Fabre. J. A., McQuillen, J. B., and Vernon, R. Gas-Liquid Flow at Microgravity Conditions: Flow Pattern and Their Transitions. Int. J. Multiphase Flow. 14. 389400 (1988).

Eastman, R. E., Feldmanis, C. J., Haskin, W. L.. and Wealver, K. L. Two-Phase Fluid Thermal Transport for Spacecraft, AFWAL-TR $843028(1984)$.

Fabre. J.. and Linè, A. Modelling of Two Phase Slug Flow. Annu. Rer. Fluid Mech. 24, 2146 (1992).

Huckerby, S. C., and Rezkallah. S. K. Flow Pattern Observations in Two-Phase Gas Liquid Flow in a Straight Tube Under Normal and Microgravity Conditions. Proc. National Heat Transfer Comference, San Diego, CA, July 1992, American Institute of Chemical Engineers (1992).

Kamp. A.. Colin, C., and Fabre, J. Bubbly Flow in a Pipe: Influence of Gravity Upon Void and Velocity Distributions, Proce of the 3rd World Conference on Experimental Heat Transfer. Flaid Mechanics and Thermodyamics, Honolulu. USA, 1418 1424. October (1993).

Lee. D. Thermohydralic and flow regime analysis for eondensing two-phase flow in a microgranity entironment. Ph. D Dissertation, Texas. A \& M University (1987).

Nicklin, D. J.. Wilkes, J. O., and Davidson, J. F. Two Phase Flow in Vertical Tubes. Trans. Inst. Chem. Engs. 40.61 68 (1962).

Reddy Karri. S. B., and Mathur. V. K. Two-Phase Flow Regime Map Predictions Under Microgravity AIChEJ., 34. $137 \quad 139(1988)$.

Reinarts, T. R. Adiahatic two phase fow regime data and modeling for zero and reduced (horizomal) acceleration fields. PhD dissertation. Univ. of Texas A \& M (1993).

Taitel, A. I., and Dukler, A. E. A Model for Predicting Flow Regime Transitions in Horizontal and Near Horizontal Gas-Liquid flow. AlChE J.,22.4755(1976).

Taitel, Y.. Barnca, D.. and Dukler. A. E. Modeling Flow Pattern Transitions for Steady L pward GasLiquid Flow in Vertical Tubes. AIChE J.. 26, 345 -354 (1980).

Wallis. G. B. One-Dimensional Two-Phase' Flow. New-York. McGraw-Hill (1969).

Weisman, J., Duncan, D., Gibson, J., and Crawford. T. Effects of Fluid Properties and Pipe Diameter on Two-Phase Flow Patterns in Horizontal Lines, Int. J. Multiphase Flow, 5, 437 462 (1979).

Weisman. J., and Kang. S. Y. Flow Pattern Transitions in Vertical and Upwardly Inclined Tubes, Int. $J$. Multiphase Flow. 7 (1981)

Zhao, L., and Rerkallah. K.S. Gas Liquid Flow Patterns at Microgravity Conditions. Int. J. Multiphase Flow. 19.751 763 (1993).

Zhao, L.. and Rezkallah. K. S. Pressure Drop in Two-Phase Annular Flow at Microgravity Conditions. AIAA 3lst Aerospace Me'ting (1993).

Zhao, L., and Rezkallah. K. S. Gas-Liquid Pressure Drop at Microgravity Conditions, Procededing of the Tenth International Heat Transfer Conference. Brighton, UK, 6. 301306 August (1994).

Zuber, N. and Findlay. J. A. Average Volumetric Concentration in Two-Phase Flow Systems. J. Heat Transfer. 87, $45368(1965)$. 



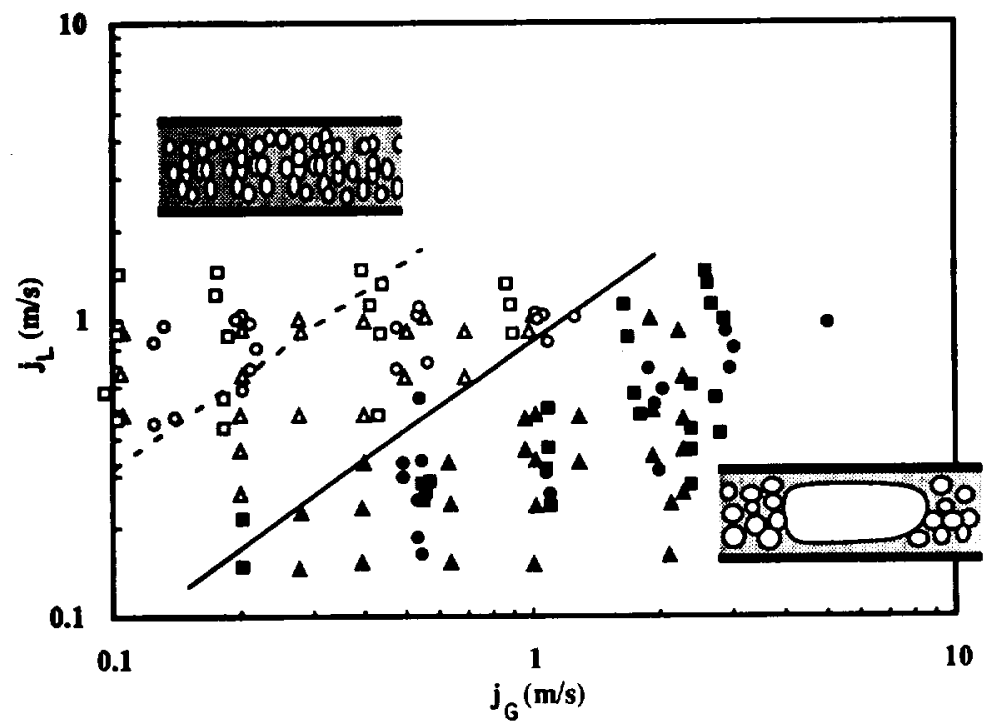

FIGURE $20^{-}$g flow pattern map in $40 \mathrm{~mm}$ dia. tube $($ Colin ef al. 1991): Data o $D=40 \mathrm{~mm}$ : $[$ bubhle, - slug flows. Transitions: bubbly to slug flow Equation (2) $x_{i}=0.2$ Equation (2) $\alpha_{c}=0.45$.

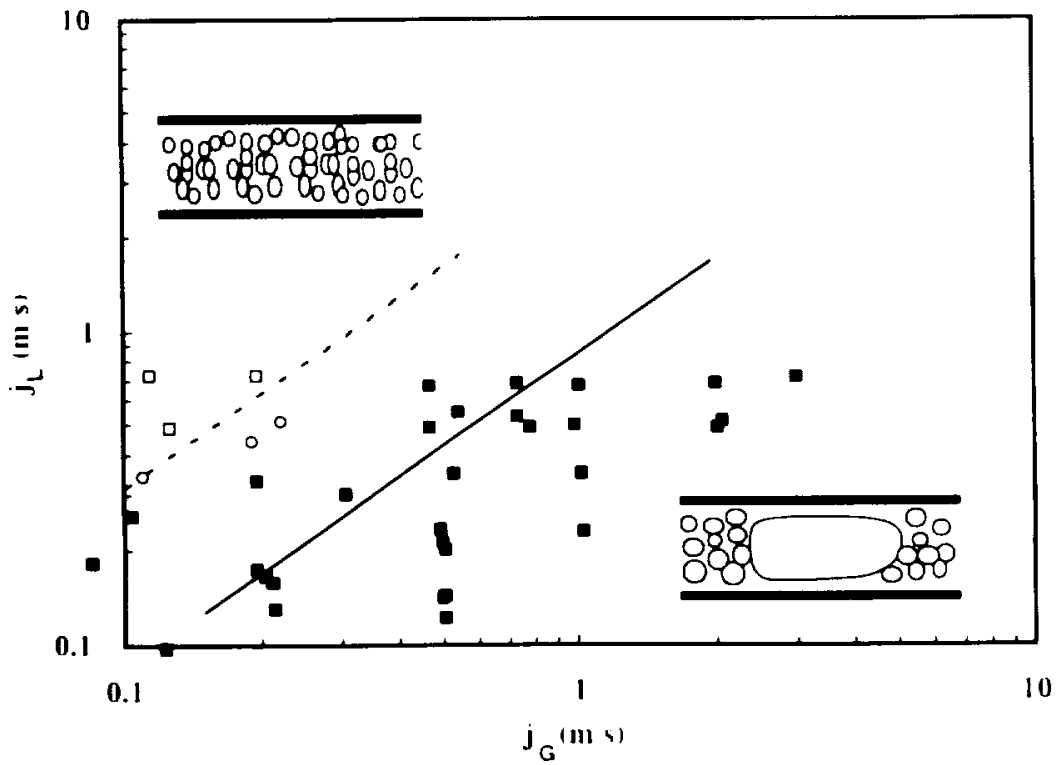

FIGURE 3 0-g flow pattern map in $25.4 \mathrm{~mm}$ dia. tube (Bousman, 1995): Data $/ \square$ bubble. $\mathbf{a}$ slug flows. o bubble to slug transition. Transitions: hubbly to slug flow: Fquation (2) $x_{i}=0.2$ Equation (2) $x_{r}=0.45$. 
different approaches. In the first one, Eastman et al. (1984), Reddy Karri and Mathur (1988), proposed to extend to 0-g conditions the correlations used in horizontal flow by Weisman et al. (1979) and Taitel and Dukler (1976) or in vertical flow by Weisman and Kang (1981) and Taitel et al. (1980). For this approach, the magnitude of the gravity vector $g$ is replaced by the value of the residual acceleration obtained in microgravity experiments. But some of these laws are inconsistent when $g$ becomes very small, In a second approach, Lee (1987) and Reinarts (1993) evaluated the different forces acting on the mixture for each flow pattern. The transition from bubble to slug flow is assumed to occur when the surface tension force becomes greater than the shear stress in the liquid phase. In the third approach, Dukler et al. (1988), Colin et al. (1991) used mechanistic models for each flow pattern to predict the transitions.

The basic mechanism which controls the transition from bubbly to slug flow is the coalescence between bubbles. The rate of coalescence depends upon the number of collisions which is related to the bubble packing. Duckler et al. (1988) pointed out that the void fraction of monodispersed bubbles at minimum packing cannot exceed 0.53 . They found that the transition occurs at a critical void fraction $\alpha_{c}$ of about 0.45 for data sets $a$ and $b$. (Table I). Assuming that in microgravity, water and air are flowing at the same average velocity $\left(U_{L} \approx U_{G}\right.$ with $U_{L}=j_{L} /(1-\alpha)$ and $\left.U_{G}=j_{G} / \alpha\right)$ they proposed a relation between $j_{G}$ and $j_{L}$. Colin et al. (1991) ignored this assumption and used a drift-flux relationship to determine the gas velocity with respect to the mixture:

$$
U_{G}=C_{0}\left(j_{G}+j_{L}\right)
$$

An experimental value of 1.2 was found for $C_{0}$ in bubbly and slug flow. With the use of Equation 1, the transition may be expressed as:

$$
j_{L}=j_{G} \frac{1-C_{0} x_{c}}{C_{0} x_{c}}
$$

For air-water flow in tube with inside diameter of 6,10 and $19 \mathrm{~mm}$ (Fig. 1), the transition occurs for a critical value of void fraction $\alpha_{c}$ equal to 0.45 , as found by other authors (Table I). But in $40 \mathrm{~mm}$ (Fig. 2) and $25.4 \mathrm{~mm}$ diameter tube (Fig. 3), the transition takes place for a critical value $\alpha_{c}$ of about 0.2 . Thus for air-water flow, the critical void fraction $\alpha_{c}$ depends on the diameter and drops in the range $D=19-25 \mathrm{~mm}$. What happens if the fluid properties are changed? The experiments of Reinarts (1993) with Refrigerant 12 in a tube of $10.5 \mathrm{~mm}$ dia., show that the critical void fraction is near 0.2 (Table I). This proves that the mechanism of coalescence leading to the transition from bubbly to slug flow is influenced by the tube diameter and by the fluid properties, the refrigerant $R 12$ having smaller viscosity and surface tension than water. Increasing the diameter and decreasing the viscosity seems to promote the formation of the large bubbles. This would normally lead to the conclusion that coalescence is promoted when the Reynolds number of the liquid $R e_{L}=j_{L} D / v_{L}$ increases. However data collected in the range $\mathrm{Re}_{\mathrm{L}}=7000-20000$ in different tube diameters do not support this idea. For same Reynolds numbers range, the transition occurs for $\alpha_{c} \approx 0.45$ in small tube whereas it occurs for $\alpha_{c} \approx 0.2$ in $40 \mathrm{~mm}$ and $25.4 \mathrm{~mm}$ diameter tube, suggesting that the transition depends of neither $j_{L}$ nor $j_{G}$. If we accept this conclusion, the value of $\alpha_{c}$ would depend on a dimensionless number which does not involve the superficial 
velocities. A good candidate is built with the fluid properties, i.e. the liquid viscosity $v_{L}$. the liquid density $\rho_{L}$ and the surface tension $\sigma$ :

$$
N_{D}=\frac{\sigma D}{\rho_{L} v_{L}^{2}}
$$

$N_{D}$ is also equal to $R e_{L}^{2} / W e$, where $W e$ is a Weber number defined as $W e=\rho_{L} j_{L}^{2} D / \sigma$. It is thus suggested that the transitional void fraction $\alpha_{c}$ be a function of $N_{D}: x_{c}=f\left(N_{D}\right)$.

This transition was checked against the existing data in Table I. However, as the void fraction is missing in some of them, $x$ was replaced by the volumetric quality of the gas, defined as:

$$
x=\frac{j_{G}}{j_{L}+j_{G}} .
$$

In bubbly flow, Equation 1 holds so that it is always possible to express the transitional void fraction $x_{c}$ versus the transitional quality $x_{c}$ :

$$
x_{c}=C_{0} x_{c} .
$$

Thus we may look for the existence of a relationship of the form $x_{\mathrm{c}}=g\left(N_{D}\right)$.

The range of variation of $x_{c}$ at the transition is plotted in Figure 4 , for different sets of data listed in Table I. Note that each data set is represented by a vertical segment, since for each set, $N_{D}$ is constant. This segment delineates the range within which a transitional regime has been found at different velocities: it may be interpreted either as the range of uncertainty of the observations or as the result of a small variation of $x_{c}$ with the mixture velocity. Excepted for the data of Huckerby et al., the values of $x_{c}$ fall

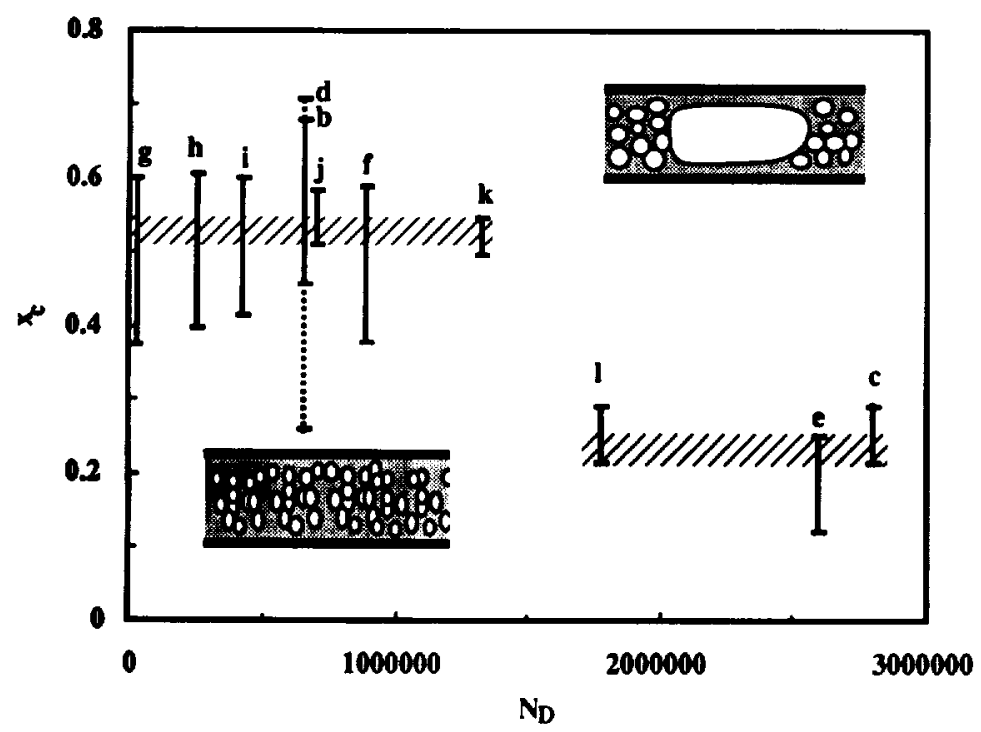

FIGURE $4 \quad 0-\mathrm{g}$ dimensionless flow pattern map: $\because-7$ transition. 
mostly within the range $0.44-0.54$, corresponding to $\alpha_{c}$ between 0.37 and 0.45 , for $N_{D}$ less than $1.3 \times 10^{6}$. For higher values of $N_{b}, x_{c}$ ranges between 0.22 and 0.27 .

Despite the uncertainty in determining the transition, two different regimes are put into light. For values of $N_{D}$ below $1.5 \times 10^{6}$, coalescence seems hindered and the transition from bubbly to slug flow appears sensitive to the bubble packing at about 0.45 of void fraction, whereas for values of $N_{D}$ greater than $1.7 \times 10^{6}$, the mechanism of coalescence is promoted and leads to a rapid transition to slug flow at $\alpha_{c}$ equal to 0.2 . An illustration is given in Figure 5 and 6 with the pictures of bubbly flow at both inlet and outlet of the tube for the same flow conditions and different tube diameters. For the smaller tube (Fig. 5) it appears clearly that the flow pattern does not change drastically between inlet and outlet. For the larger tube (Fig. 6) big bubbles are observed at the outlet indicating that the collisions between bubbles were very effective in promoting coalescence.

The available results for the transition from bubbly to slug flow may be summarized as follows:

$$
\begin{aligned}
& \frac{\sigma D}{\rho_{L} v_{L}^{2}}<1.5 \times 10^{6} \Rightarrow x_{c} \approx 0.45, \quad x_{c} \approx 0.54 \\
& \frac{\sigma D}{\rho_{L} v_{L}^{2}}>1.7 \times 10^{6} \Rightarrow x_{c} \approx 0.20, \quad x_{c} \approx 0.24
\end{aligned}
$$

This relationship has the advantage to take into account the fluid properties in the evolution from bubbly to slug flow, - evolution being here more appropriate than
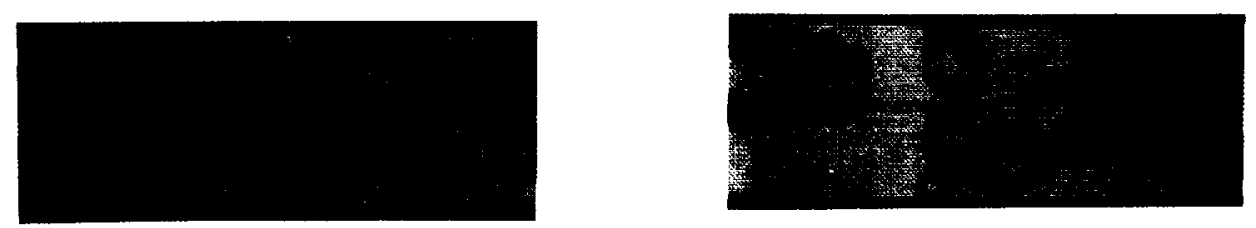

FIGURE 5 Flow visualization in $10 \mathrm{~mm}$ dia. tube for $j_{L}=0.46 \mathrm{~m} / \mathrm{s}, j_{G}=0.11 \mathrm{~m} / \mathrm{s}$. Left: inlet test section. Right: outlet test section (80 D downstream).
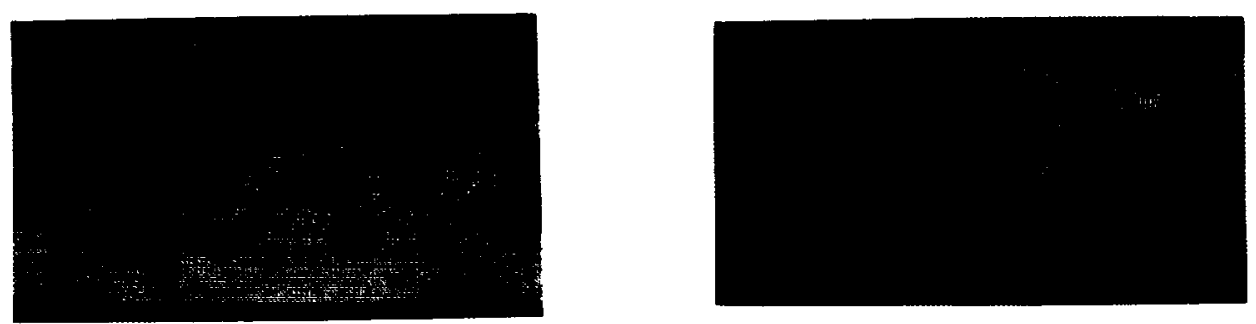

FIGURE 6 Flow visualization in $40 \mathrm{~mm}$ dia. tube: $j_{L}=0.41 \mathrm{~m} / \mathrm{s}, j_{\mathrm{G}}=0.11 \mathrm{~m} / \mathrm{s}$. Left: inlet test section. Right: outlet test section (80 D downstream). 
transition which evokes a bifurcation in flow pattern-. Equation 6 raises an interesting question: is there a transition between the hindering-coalescence regime and the promoting-coalescence regime? The existence of such a transition has to be confirmed by additional experiments in the range of $N_{D}$ between $1.5 \times 10^{6}$ and $1.7 \times 10^{6}$. It also has to be explained based on physical arguments.

Due to the lack of experimental results these conclusions are still questionable. They suggest however the line to follow for future experiments, concerning the transition. With small tube, experiments without phase change, the gap will be difficult to fill. Indeed the only way would be to reduce the viscosity, but that would use fluids as alcohols or solvent, which would conflict with aircraft safety restrictions. In contrast experiments in large tubes will be highly valuable since the viscosity can be easily increased by addition of glycerine in water and the surface tension can be reduced with the use of mineral oil.

\section{PHASE FRACTION}

Several authors have published data concerning either void fraction or average gas velocity. Different methods have been used to determine the cross-sectional averaged void fraction $\alpha$. In the studies of Colin et al. (1991) and Colin and Fabre (1995) conductance probes made of 2 stainless steel flush-mounted electrodes inside the tube wall were used. Bousman and Dukler (1993) have used conductance probes with two parallel wire in order to determine the void fraction in the bubbly region of bubbly and slug flow and the film thickness in the separated flow region of slug and annular flow. The cross-sectional averaged gas velocity $U_{G}$ was determined from flow pictures (Colin and Fabre, 1995) or by cross correlating the signals from two pairs of wire positioned along the tube axis (Bousman and Dukler, 1993). The measured values of gas velocities $U_{G}$ were compared to those calculated from the experimental void fraction $U_{G}=j_{G} / x$ (Colin and Fabre, 1995). In most of the cases a good agreement is found for bubbly flow, in contrast to slug flow for which some discrepancies have been observed in small tubes. This is partly due to the difficulty to calibrating the conductance probes in small tubes, since the apparent electrical conductance is strongly sensitive to the local void distribution inside the tube, i.e. to the flow pattern.

The gas velocity $U_{G}$ is plotted versus the mixture velocity $j=j_{G}+j_{L}$, in Figure 7 for bubbly flow and in Figure 8 for slug flow. In Bousman and Dukler's results $U_{G}$ was determined from the cross correlation between the signals of two void fraction probes located at certain distance from each other whereas in our present results, $U_{G}$ was obtained from an image processing method. These results lead to the following conclusions: as $C_{0}>1$ there is an average drift of the gas with respect to the mixture equal to $U_{G}-j=\left(C_{0}-1\right) j$; regarding the drift, bubbly and slug flow can be hardly distinguished. This drift is reasonably well predicted by Equation 1, as already proposed by Colin et al. (1991):

$$
U_{G}=C_{0} j \text { with } C_{0} \approx 1.1-1.3 .
$$

Where does the drift come from in the absence of gravity? 


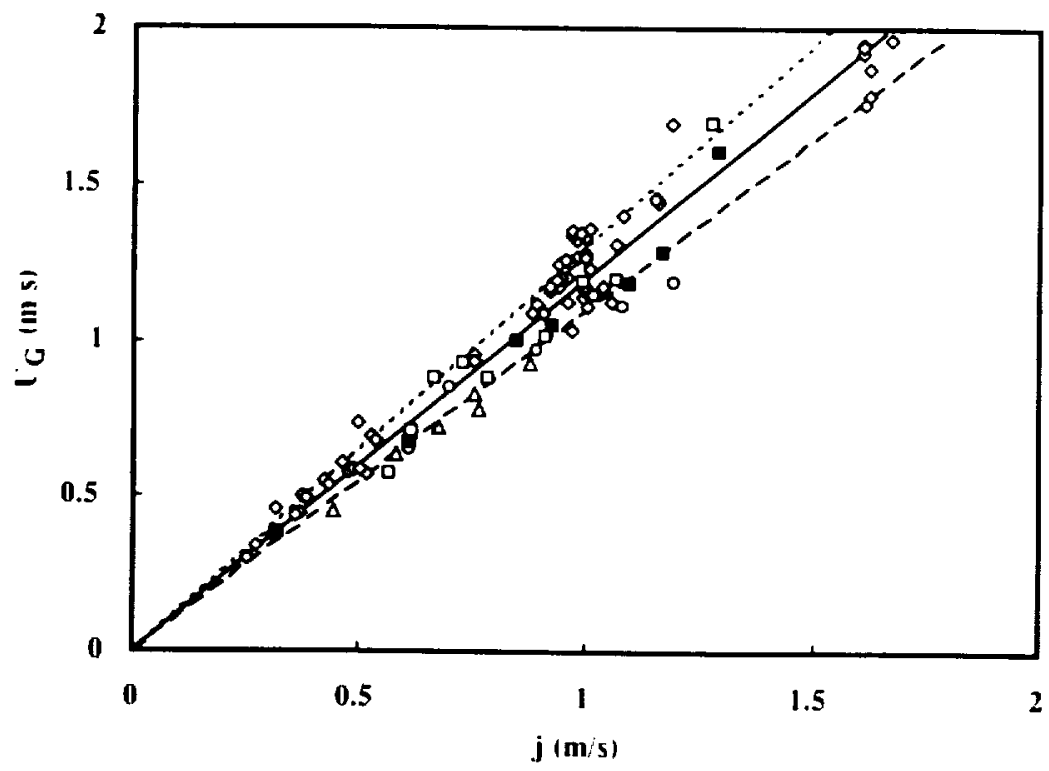

FIGURE 7 Gas velocity in air-water bubbly flow: $O$ Data i $D=6 \mathrm{~mm}$, $\square$ Data $j D=10 \mathrm{~mm}, \triangle$ Data $k D=19 \mathrm{~mm}$, Data $f D=12.7 \mathrm{~mm}$, $\diamond$ Data $c D=40 \mathrm{~mm}$, Equation (1) with $-C_{0}=1.2$; $\ldots$ $C_{0}=1.1 ; \ldots C_{0}=1.3$.

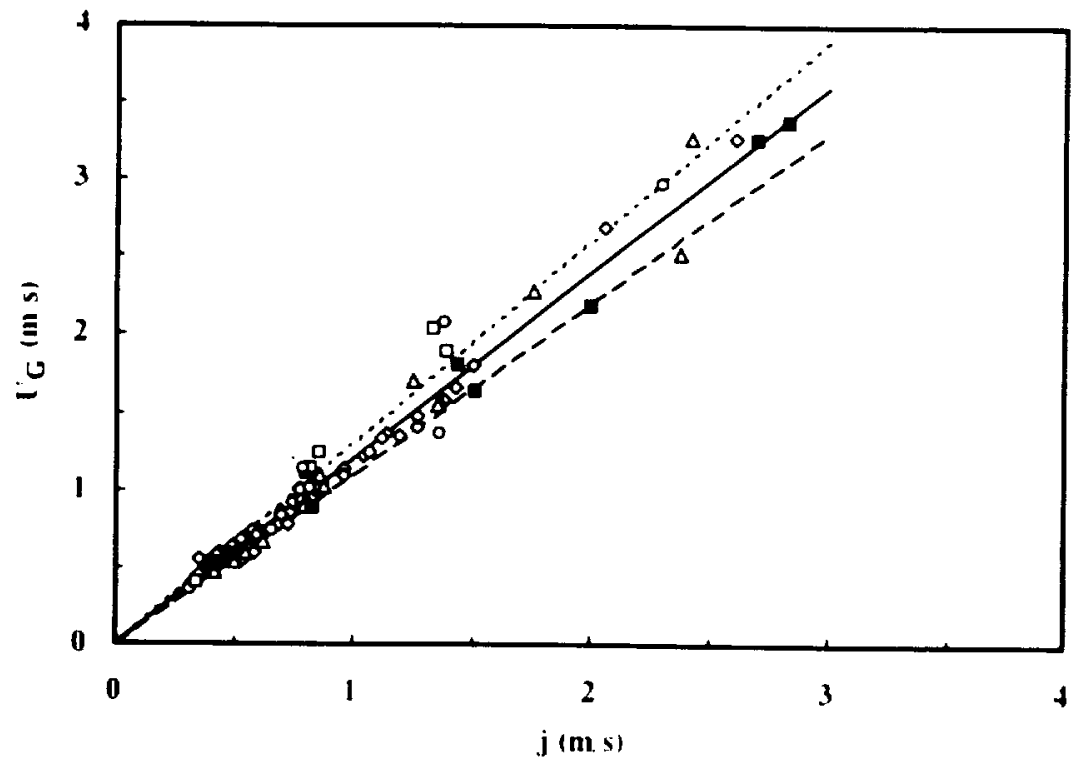

FIGURE 8 Gas velocity in air-water slug flow: Data $i \quad D=6 \mathrm{~mm}$, $\square$ Data $j D=10 \mathrm{~mm}, \triangle$ Data $k D=19 \mathrm{~mm}$, Data $f D=12.7 \mathrm{~mm}$, $\diamond$ Data $c D=40 \mathrm{~mm}$, Equation (1) with $-C_{0}=1.2 ;---C_{0}=1.1$; .... $C_{0}=1.3$. 
The local velocity difference is expected to be weak in the absence of body force. This is confirmed by the experiments of Kamp et al. (1993) whose results are plotted in Figure 9. In this figure the local liquid velociy $u_{L}$, measured with a hot film anemometer, is compared to the local gas velocity $u_{G}$ obtained from a double optical probe. The local velocity difference between phase is small: it remains within the range of uncertainty of the measurements. These results provide a strong argument that no local slip exists in microgravity, thus $u_{G} \approx u_{L} \approx u$.

Nevertheless, even in the absence of local slip, an average drift can exist if the gas is mainly concentrated in the flow regions where the velocity is the greatest. Let $\varepsilon$ be the local void fraction and $\langle-\rangle=A^{-1} \int_{A}-d A$ the averaging operator over the cross section $A$ of the tube. Then $C_{0}$ may be expressed as:

$$
C_{0}=\frac{\langle\varepsilon u\rangle}{\alpha j} .
$$

$C_{0}$ characterizes the relation between the distributions of local velocity and void fraction. Thus, the value of $C_{0}$ gives some information on the local distribution of void fraction. It is greater than unity if the radial distribution of void fraction is at its maximum at the tube axis. Kamp et al. (1993) have determined the distribution of local void fraction by using optical probes: they found indeed a maximum of the local void fraction at the tube axis (Fig. 9). Following Zuber and Findlay (1965) it is possible to determine the value of the coefficient $C_{0}$ from Equation 8. Assuming that the local

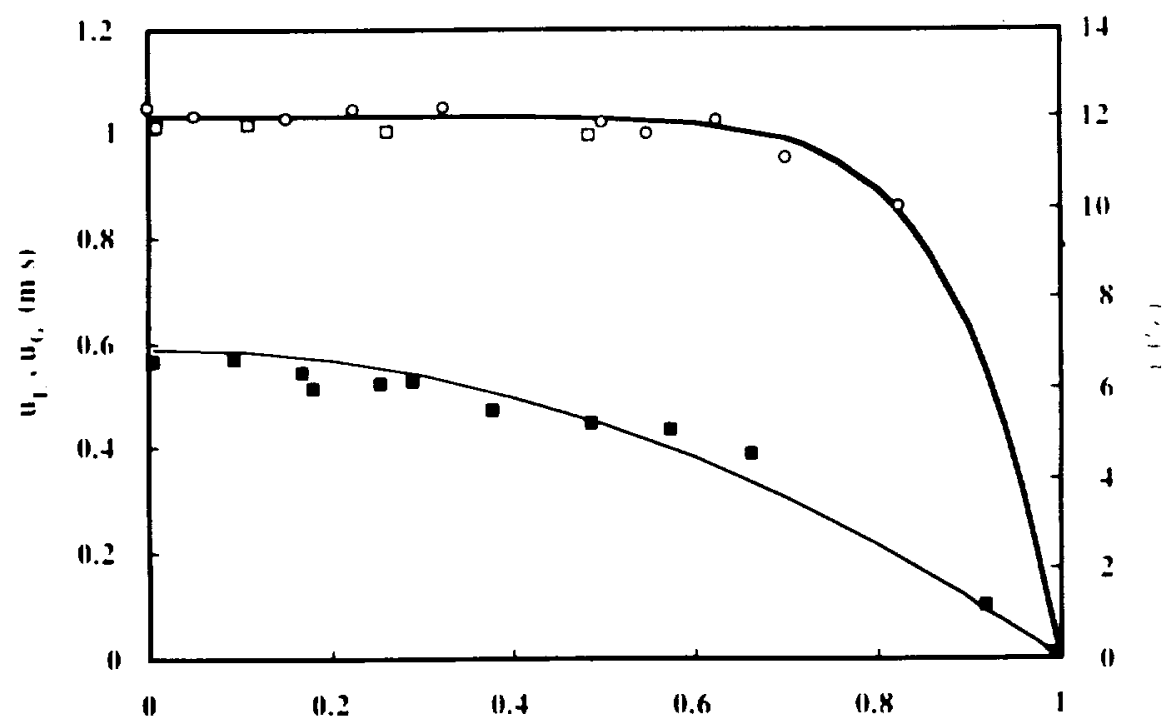

FIGURE 9 Void fraction profile and velocity profiles of liquid gas in $40 \mathrm{~mm}$ dia. tube: $J_{L}=0.77 \mathrm{~m} / \mathrm{s}$, $j_{G}=0.044 \mathrm{~m} / \mathrm{s}$. $O$ velocity of liquid, $\square$ velocity of gas, $\square$ local void fraction,

Equation (9) with $n=2$. 
velocity and void fraction distributions have the form:

$$
\begin{aligned}
& \varepsilon=\varepsilon_{0}\left[1-\left(\frac{r}{R}\right)^{n}\right] \\
& u=u_{0}\left[1-\left(\frac{r}{R}\right)^{m}\right]
\end{aligned}
$$

the coefficient $C_{0}$ becomes:

$$
C_{0}=\frac{m+n+4}{m+n+2}
$$

For the test corresponding to $j_{L}=0.77 \mathrm{~m} / \mathrm{s}$ and $j_{G}=0.044 \mathrm{~m} / \mathrm{s}$, the values of $m$ and $n$ are found to be equal to 9 and 2 respectively from using a least square fit. The resulting value for $C_{0}$ is 1.15 , in good agreement with the value calculated from the experimental void fraction given by the conductance probe.

The effect of spatial distribution does explain that there exists an average drift in bubbly flow even if there is no local slip. Therefore the key issue for bubbly flow is to understand the lateral force balance which is responsible for the concentration of void near the axis. The answer is not cleafly known at the present time and the local two-fluid models fail to predict the correct distribution. An effort has to made on this simple and fundamental case. Some elementary experiments like the motion of a single bubble in pipe at microgravity conditions would be helpful to understand the phenomenon: it could be a physical or numerical experiment. On the other hand the experimental analysis of the local distributions of velocity and void are also great help. The first experiments carried out by Kamp et al. on the determination of the local structure of the flow, show however some limitations in performing such experiments in parabolic flights because of the short duration and the poor quality of the microgravity conditions in parabolic flights. This pleads strongly for an in-orbit experiment which could be decisive.

As previously mentioned $C_{0}$ has for slug flow the same trend than for bubbly flow (Fig. 8). This was already pointed out by Colin et al. (1991), based on their experiments in $40 \mathrm{~mm}$ diameter tube; this is also confirmed by the present experiments in smaller tubes and by those of Bousman and Dukler (1993). In slug flow the gas is mostly contained in the large cylindrical bubbles. It is thus acceptable to assume that $U_{G} \approx V$, where $V$ is the velocity of the cylindrical bubbles. The result on $C_{0}$ shows that the Nicklin et al. (1962) relationship for turbulent flow $V=1.2 j+0.35(g D)^{1 / 2}$, is valid also in the limit $g=0$. From the inviscid flow theory of Collins et al. (1978), one shoul be able to predict the value of $C_{0}$ for both laminar and turbulent flows of the continuous phase in the limit $g=0$ :

$$
\begin{array}{ll}
C_{0}=2.27 & \text { for laminar flow } \\
C_{0}=\frac{\log R e_{L}+0.089}{\log R e_{L}-0.74} & \text { for turbulent flow }
\end{array}
$$

The turbulent flow relationship gives a value decreasing from 1.25 to 1.2 for $R e_{L}$ in the range $10,000-100,000$. However this variation is within the range of uncertainty of the 
measurements. On the other hand at smaller Reynolds number, typically $<5,000, C_{0}$ is likely to increase up to reach the theoretical value of 2.27 when the flow becomes laminar. In $1-g$ flow this behavior was clearly shown by Fréchou (see Fabre and Liné, 1992). Some additional experiments are thus necessary to determine the behavior of $C_{0}$ for $R e_{L}$, within the range 500-5,000. Indeed in this range, the transition -which must exist-is highly indicative of a modification of flow regime upstream the nose of the cylindrical bubble.

\section{WALL FRICTION}

In microgravity the friction at the wall is directly related to the pressure drop $d P / d x$. Indeed the mean shear stress $\tau_{w}$ may be expressed by

$$
\tau_{w}=\frac{D}{4} \frac{d P}{d x} .
$$

Measuring the pressure drop gives access to the wall shear stress. This holds for 1-g horizontal flow as well. Most of the results on pressure drop reported in the literature concerns two-phase flow at high void fraction in annular or slug pattern (Chen et al., 1991; Bousman and McQuillen, 1994; Zhao and Rezkallah, 1994). Only scarce results have been published for bubbly or slug flow.

It has already been pointed out by Chen et al. (1991) that pressure drops are generally greater in microgravity than in 1 -g horizontal flow. In order to illustrate this peculiarity, the values of $\tau_{w}$, which are calculated from different data sets (Bousman and Dukler, 1993; Colin and Fabre, 1995) have been replotted in Figures 10 and 11 for two different tube diameters. In Colin and Fabre experiments, the pressure drop is measured from two different transducers and only the results which agree within $10 \%$ are retained. The difference between $0-\mathrm{g}$ and $1 \mathrm{~g}$ g results is shown to be smaller for the smaller tube. This difference also decreases when the gas velocity increases. It is expected that, when inertial force is large enough compared to gravity, there is no reason to distinguish $0-\mathrm{g}$ flow and $1 \mathrm{-g}$ horizontal flow: this is probably true when $\rho_{G} U_{G}^{2} / \Delta \rho g D \gg 1$, i.e. in annular flow in small tubes.

What controls the wall friction in bubbly flow? One method to answer to this question goes through the analysis of the friction factor, generally defined as $f=\tau_{w / 1} /(1 / 2) \rho U^{2}$. This definition raises the following question: what density and velocity scales must be chosen? We have the choice between the liquid quantities $\rho_{L}, U_{L}$. or the mixture quantities $\rho_{M}=x_{L} \rho_{L}+x_{G} \rho_{G}, j$ or a combination of both. It is possible for example to introduce the two following definitions:

$$
\begin{gathered}
f_{M}=\frac{\tau_{w^{\prime}}}{\frac{1}{2} \rho_{M} j^{2}} \\
f_{L}=\frac{\tau_{w}}{\frac{1}{2} \rho_{L} U_{L}^{2}}
\end{gathered}
$$

The fundamental issue is not only a matter of definition, it is intimately related to the physics of momentum transfer at the wall. A piece of the answer can be given from 


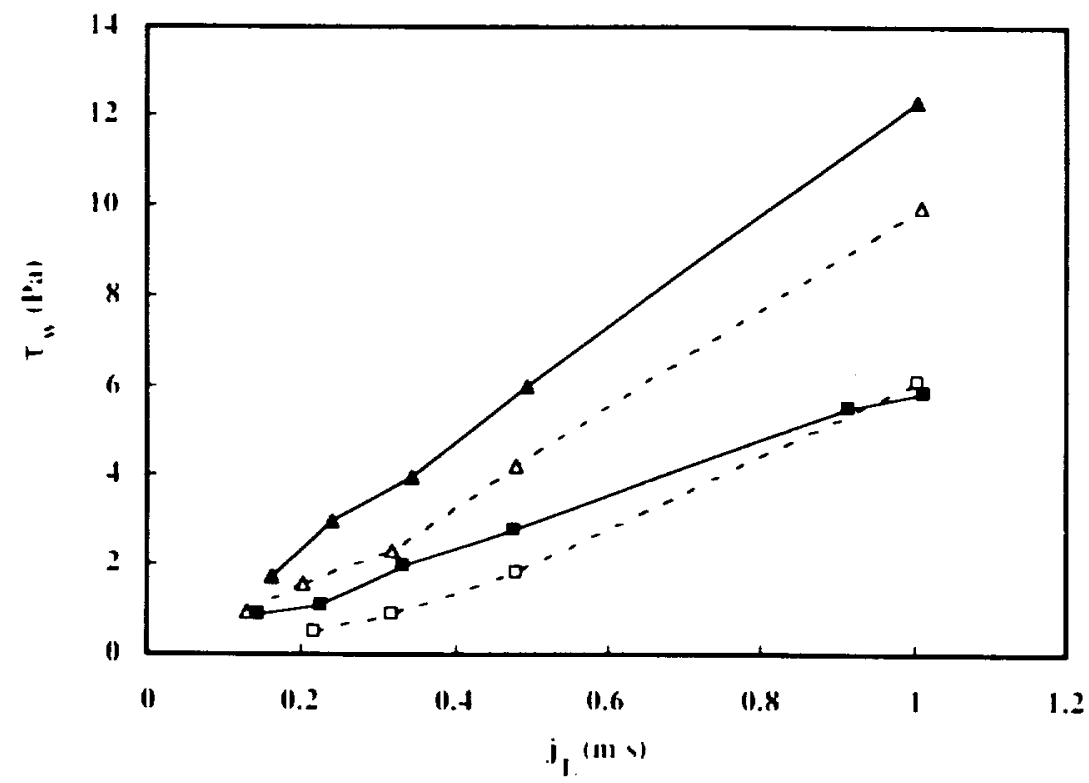

FIGURE 10 Wall shear stress in $0-\mathrm{g}$ and $1-\mathrm{g}$ in $19 \mathrm{~mm}$ tube: $0-\mathrm{g}$ flow: $\square j_{G}=0.2 \mathrm{~m} / \mathrm{s}, \Delta j_{G}=2 \mathrm{~m} / \mathrm{s}, 1-\mathrm{g}$ horizontal flow: $j_{G}=0.2 \mathrm{~m} / \mathrm{s} \wedge j_{G}=2 \mathrm{~m} / \mathrm{s}$.

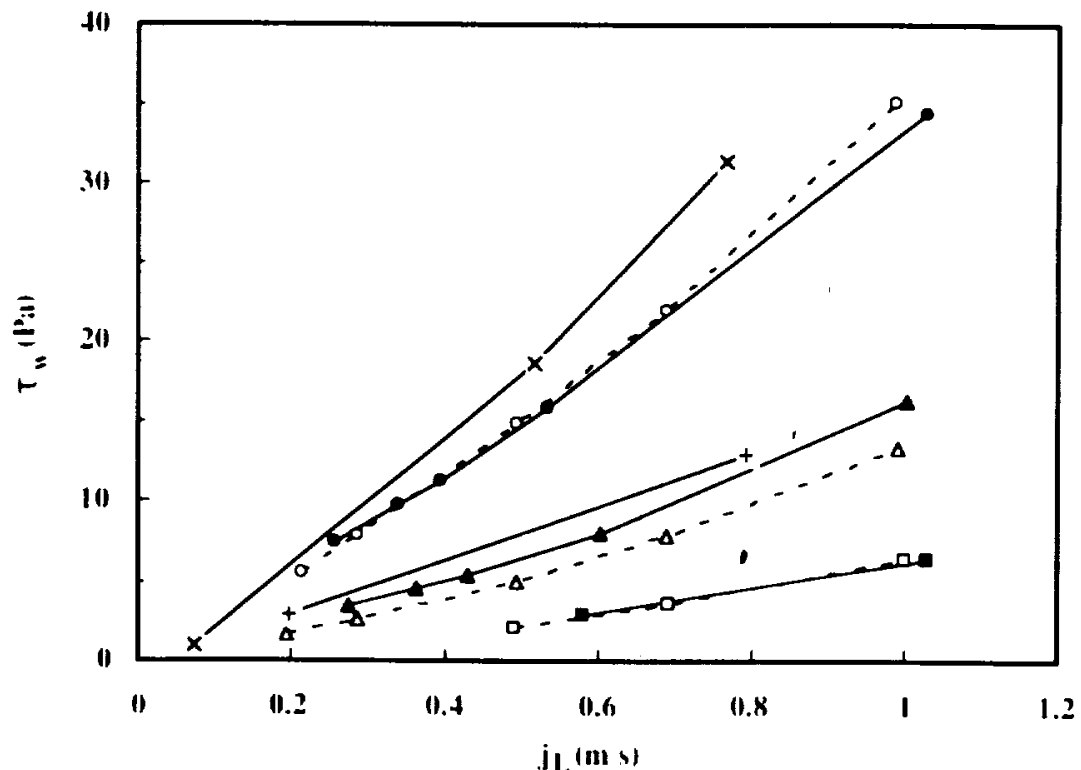

FIGURE 11 Wall shear stress in $0-\mathrm{g}$ and $1-\mathrm{g}$ in 10 and $12.7 \mathrm{~mm}$ tube: $0-\mathrm{g}$ flow: $D=10 \mathrm{~mm}: \square j_{G}=0.2 \mathrm{~m} / \mathrm{s}$, $\Delta j_{G}=2 \mathrm{~m} / \mathrm{s}, O j_{G}=9.5 \mathrm{~m} / \mathrm{s}, D=12.7 \mathrm{~mm}:+j_{G}=2 \mathrm{~m} / \mathrm{s}, \times j_{G}=10 \mathrm{~m} / \mathrm{s} 1-\mathrm{g}$ horizontal flow: $D=10 \mathrm{~mm}$, $j_{G}=0.2 \mathrm{~m} / \mathrm{s}, \Delta j_{G}=2 \mathrm{~m} / \mathrm{s}, \bullet j_{G}=9.5 \mathrm{~m} / \mathrm{s}$. 


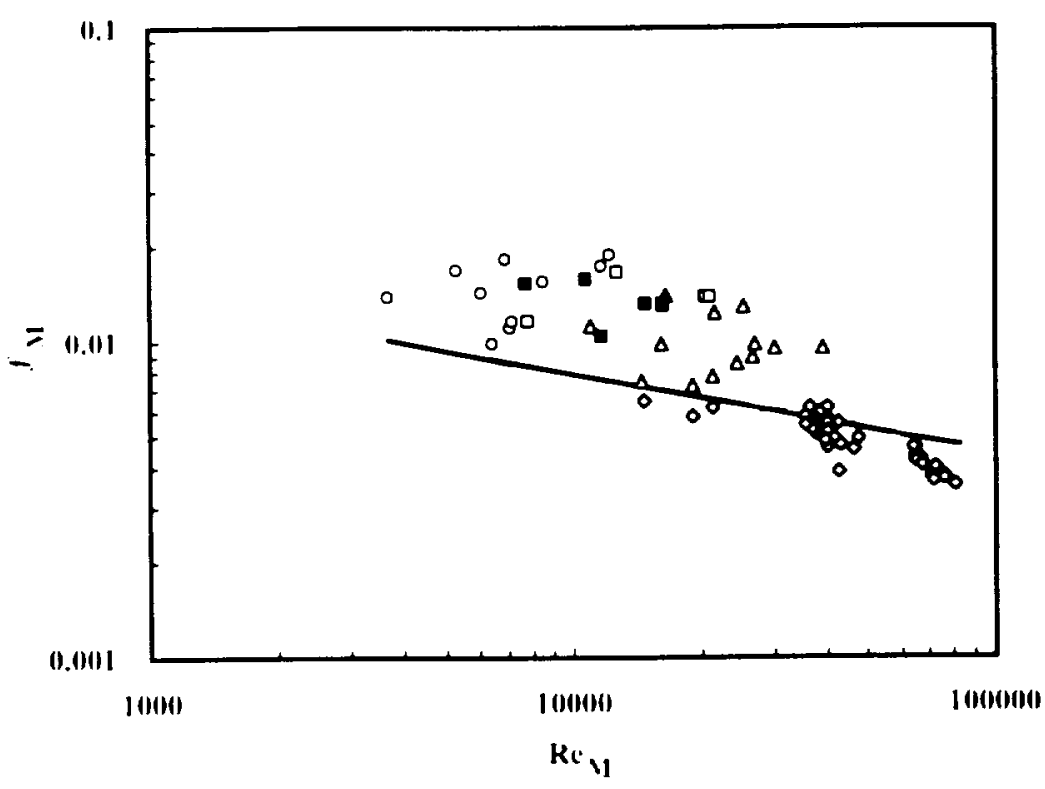

FIGURE 12 Wall friction factors $f_{M}$ in bubbly flow: $0-\mathrm{g}$ flow $O D=6 \mathrm{~mm}, \square D=10 \mathrm{~mm}, \triangle D=19 \mathrm{~mm}$, - $D=12.7 \mathrm{~mm}, \diamond D=40 \mathrm{~mm}$, Blasius relationship

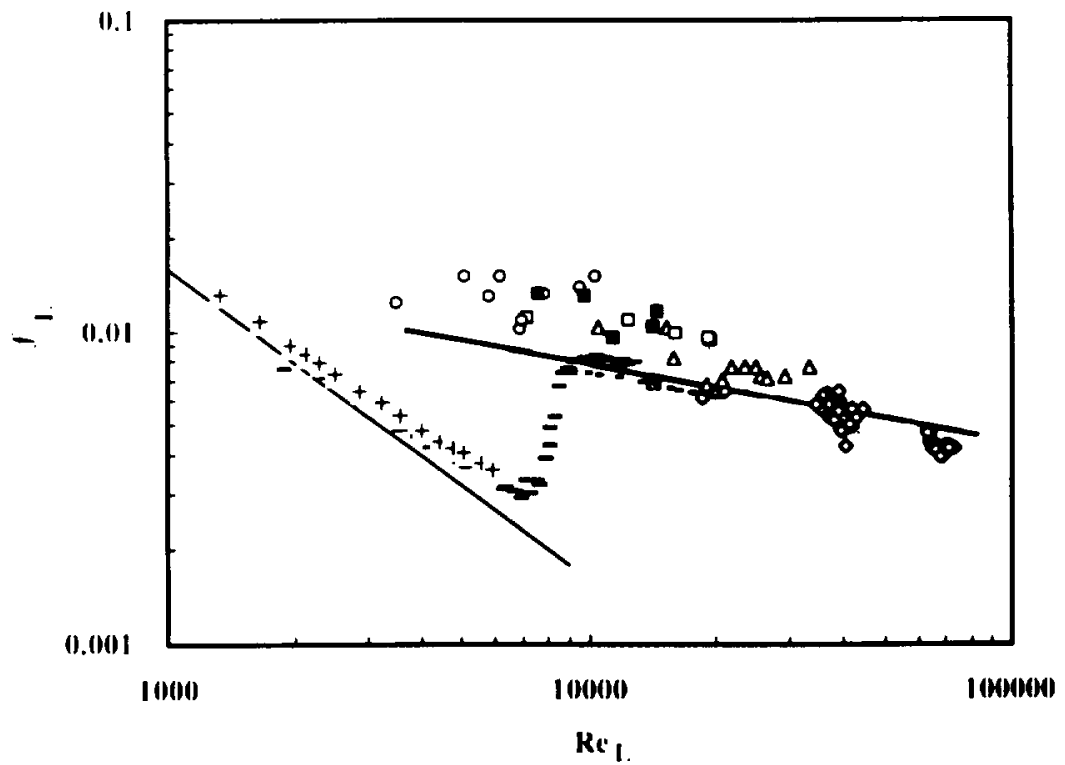

FIGURE 13 Wall friction factors $f_{L}$ in bubbly flow: $0-\mathrm{g}$ flow $O D=6 \mathrm{~mm}, \square D=10 \mathrm{~mm}, \triangle D=19 \mathrm{~mm}$, - $D=12.7 \mathrm{~mm}, \diamond D=40 \mathrm{~mm}$ single-phase flow: $+D=6 \mathrm{~mm}-D=10 \mathrm{~mm}-D=19 \mathrm{~mm}$, Blasius, Poiseuille relationship. 
intuitive arguments: as the void is mainly concentrated at the tube axis, the momentum transfer at the wall must be mainly controlled by the liquid motion. It is thus anticipated that the friction factor defined by Equation 14 be given under the form $f_{L}=F\left(R e_{L}, \alpha\right)$, where $f_{L}=F\left(R e_{L}, 0\right)$ is the single phase flow relationship. An alternative is to express the friction factor defined by Equation 15 as $f_{M}=F\left(R e_{M}, x\right)$. The experimental results are plotted in Figures 12 and 13 with the two choices of dimensionless variables: the results are less scattered in Figure 13, justifying that the liquid motion controls the shear stress at the wall. In addition, the experimental points are not too far from the single phase Blasius relationship:

$$
f_{L}=0.079 R e_{L}^{-0.25}
$$

This relationship may be used with confidence to predict the pressure drop in the range $R e_{L}=20,000-80,000$. Indeed for bubbly flow in $40 \mathrm{~mm}$ diameter tube, it has been shown by Kamp et al. (1993) at $R e_{L}=40,000$, that the near wall velocity profiles follow the same logarithmic law as far single-phase flow: the presence of the bubbles does not significantly modify the local structure of the flow. In contrast, for Reynolds number less than 20,000, the experimental friction factor is greater than predicted by Equation (16). This tendency may be explained as follows: as the Reynolds number decreases, the thickness of the viscous layer increases but the presence of large bubbles may affect this layer near the tube wall. This is due to an additional production of turbulence induced by the distortion of the liquid flow by the bubble motion: this extra turbulence has indeed been put into light in bubbly flow at microgravity conditions by Kamp et al. (1993).

Another interesting result which appears in Figure 13 concerns the transition between laminar and turbulent regime. Single phase flow experiments have been carried out in the same loop. The corresponding friction factor has been added in Figure 13. For experiments in 6 and $10 \mathrm{~mm}$ tube, the Reynolds number ranges between 1,000 and 40,000 . In the $6 \mathrm{~mm}$ diameter tube, when the single-phase flow is laminar, the wall friction factor follows the theoretical Poiseuille relationship $f_{L}=16 / R e_{L}$. The transition from laminar to turbulent flow is observed at $R e \approx 8,000$ in single-phase flow in the $10 \mathrm{~mm}$ diameter tube, the jump of $f_{L}$ near $R e_{L}=8,000$ being the signature of this transition. Clearly two-phase bubbly flow does not display the same behavior at least for $R e_{L}>4,000$. There are some interesting and yet unresolved questions related to the wall friction behavior at smaller Reynolds number. Does a transition exist between laminar and turbulent flow and does the wall friction decrease at smaller Reynolds numbers? This motivate the needs for careful experiments at low Reynolds number.

Let us now discuss the case of slug flow from the experimental results plotted in Figure 14. In comparison to bubbly flow the same trends are observed, although the experimental data are much more scattered. There is a fundamental difference between bubbly and slug flow: the intermittence. In a reference that moves with the long cylindrical bubbles, the flow appears almost frozen in microgravity. Indeed each long bubble is separated by bubbly liquid slugs in which the small bubles move at about the same velocity as the long ones. By using the unit cell concept proposed by Wallis (1969), the pressure drop may be written under the form (see for example Dukler and Fabre, 1992):

$$
\frac{d P}{d x}=\frac{\beta\left(\tau_{L w B} S_{L w B}+\tau_{G w B} S_{G w B}\right)+(1-\beta)\left(\tau_{L w S} S_{L w S}\right)}{A}
$$




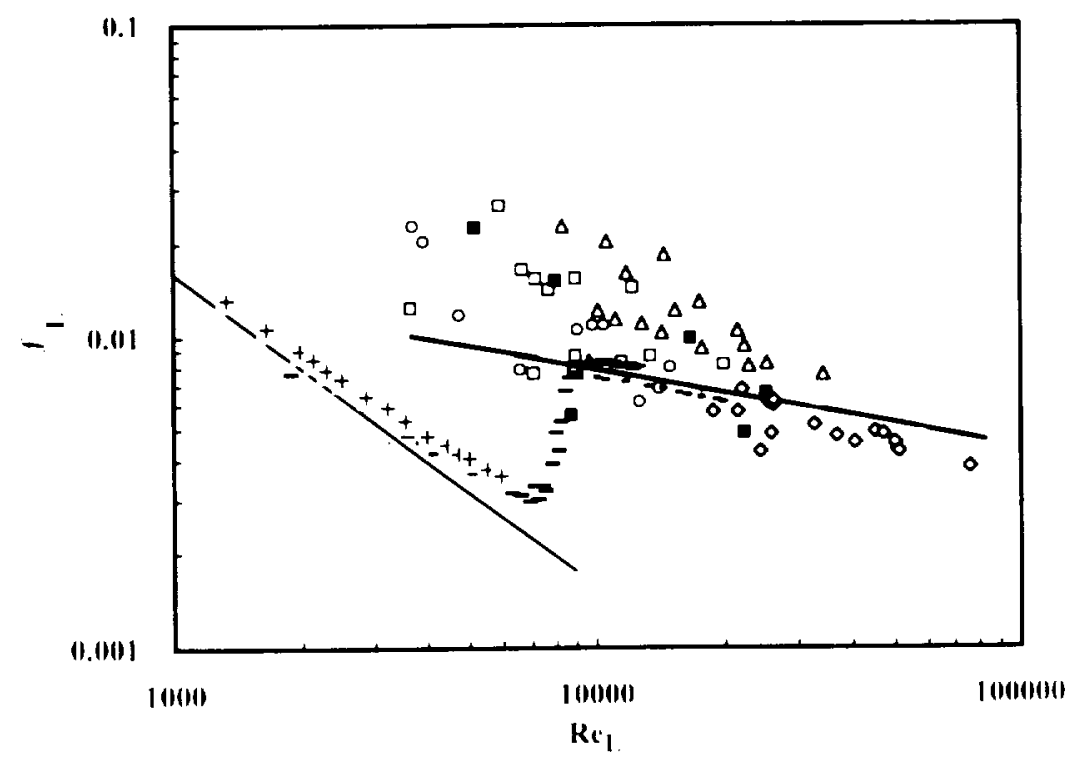

FIGURE 14 Wall friction factors $f_{L}$ in slug flow: 0 -g flow $\bigcirc D=6 \mathrm{~mm}, \square D=10 \mathrm{~mm}, \triangle D=19 \mathrm{~mm}$, - $D=12.7 \mathrm{~mm}, \diamond D=40 \mathrm{~mm}$ single-phase flow: $+D=6 \mathrm{~mm},-D=10 \mathrm{~mm},-D=19 \mathrm{~mm}, \square$ Blasius, Poiseuille relationship.

where the subscripts $B$ and $S$ stand for the long bubble and slug regions respectively, $A$ is the crossed sectional area, $S$ is the wetted perimeter and $\beta$ the ratio of the long bubble length to the total cell length. As the gas does not wet or contact the wall in microgravity, the mean shear stress defined by Equation 13 becomes:

$$
\tau_{w}=\beta \tau_{w S}+(1-\beta) \tau_{w D}
$$

For Equations 17 and 18 to be written under this form one has to accept that the flow is fully developed in both regions. In the absence of gravity, there is no driving force to move the liquid film around the long cylindrical bubbles: this is verified from video sequences, the liquid velocity being estimated from the motion of tiny bubbles in the liquid film around the long bubbles. Thus the shear stress in the long bubbles is probably weak in contrast to this in the liquid slugs. Consequently it may be assumed that $\tau_{w} \approx(1-\beta) \tau_{w D}$. With the fully developed flow assumption, the velocity of the mixture in the liquid slugs is equal to $j$. If we accept that the friction factor in bubbly slugs is given by Equation 16, as shown by the foregoing results, then the shear stress in slug flow must be equal to the shear stress in the corresponding bubbly flow, times $1-\beta$. This assumption would lead to the result that the shear stress in slug flow must be smaller than the shear stress in the corresponding bubbly flow. This is not supported by the experimental results which have shown the opposite. To arrive to this paradox we have used two assumptions: the flow in each region is fully developed and the friction in the liquid film is weak enough to be disregarded. It is likely that the second assumption is correct. This is not the case for the first assumption since the slugs are short and the 
velocity profile can hardly be considered as fully developed in the moving frame. This assumption has to be reconsidered if we want to correctly predict the friction at the wall in slug flow.

\section{CONCLUSION}

During the past decade, a considerable effort has been done to understand two-phase flow at microgravity conditions. The studies have generally concerned flow pattern transitions and at a least extend void distribution and pressure drop. Focusing our attention on bubbly and slug flows, we attempted to present the state of our current knowledge from the current available data. Some conclusions may be drawn for the future research.

Concerning the transition from bubble to slug flow, we suggest the existence of a possible transition for some value of $\sigma D / \rho_{L} v_{l}^{2}$ between $1.5 \times 10^{6}$ and $1.7 \times 10^{6}$. Future experiments have to be carried out to confirm this suggestion. Moreover this transition poses the delicate problem of predicting bubble coalescence in flow conditions, which must be analysed from this perspective.

The void fraction may be predicted with an acceptable accuracy from Equation 1. This equation proves that a mean drift between gas and liquid exists. For bubbly flow, the origin of the drift must be found in the concentration of void at the tube axis. At the present time the force balance which controls the bubble migration towards the axis remains unclear and specific experiments are needed. For slug flow the motion of long bubbles is well explained by the theory in the limit $g=0$. However some experiments are needed at lower Reynolds numbers, say between 500 and 5,000 to clarify this motion.

At microgravity conditions the pressure drop is the direct signature of the mean shear stress at the wall. In bubbly flow, the single-phase flow relation of Blasius is acceptable although there is a tendency to underestimate the friction when $R e_{L}<$ 40,000 . The single-phase relationship ignores the presence of bubbles which needs to be taken into account. Some new insight into the local mechanism of bubble turbulence interaction is needed. For slug flow, the friction is not predicted with a sufficient accuracy from the unit cell concept: the fully developed flow assumption has to be reconsidered. Last but not least, the evolution of the friction factor with the Reynolds number may indicate whether a transition from laminar to turbulent flow exists in two-phase flow. In this view, the analysis of the velocity fluctuations may be useless because the bubbles induce small velocity fluctuations by their motion relative to the wall that may be difficult to discriminate from turbulence fluctuations.

\section{ACKNOWLEDGEMENTS}

The study of two-phase flow at microgravity conditions was initiated by A. E. Dukler to whom we are greately indebted. This paper is the modest tribute that we would like to pay to his memory. The cooperation which followed these discussions put altogether the efforts of the "Institut National Polytecnique de Toulouse" and the University of Houston. Most of the results quoted in this paer testify to this scientific cooperation. The experimental work was strongly supported by CNES, ESA and NASA. 